\title{
COMPARISON OF CONVENTIONAL SLAB AND METAL DECK COMPOSITE SLAB METHOD TO TIME AND COST IN TRANSPARK CIBUBUR PROJECT
}

\author{
Anjas , Handayani \\ Faculty of Engineering \\ Mercu Buana University Jakarta, Indonesia \\ anjas_handayani@yahoo.com \\ Mochamad Elvan, Reza \\ Faculty of Engineering \\ Mercu Buana University Jakarta, Indonesia \\ elvanrz@gmail.com
}

\begin{abstract}
in the implementation of the Transpark Cibubur project, especially in Tower C Apartment there are several problems in conventional slab methods including the need for a longer construction time. With some thoughts and considerations, the work method of floor slab was changed from conventional method to metal deck composite slab method. In this research, a comparative of conventional slab and metal deck composite slab method in Tower $C$ of the Cibubur Transpark Project is the object of research to compare the two methods to speed of implementation and cost requirements. The analysis results show in Tower $C$ 16th to 19th floor for metal deck composite slab method is more expensive than conventional slab method with a cost difference Rp. 137.252.215,54. While in terms of work time, conventional slab method are longer than metal deck composite slab method with a time difference for 25 days.
\end{abstract}

keywords : Conventional Slab, Metal Deck Composite Slab, Time, Cost.

\section{INTRODUCTION}

Based on direct observations and information in the field, in the implementation of the Transpark Cibubur project, especially in Tower C Apartment using conventional floor slab method. With some thoughts and considerations, the work method of floor slab was changed from conventional method to metal deck composite slab method.

Floordeck is a galvanized material that is shaped like a zinc wave but does not function as a roof covering material. Floordeck is a coating material under cast concrete floor plates instead of wooden formwork (multiplex). Floordeck is also designed to convert the use of iron diameter and convert the thickness of concrete cast. The buckling system (plate wave) is designed to help the strength of the concrete floor cast structure.

Floordeck can reduce the volume of cast concrete by $15 \%$ to $25 \%$ and reduce the amount of steel reinforcement for concrete casts because the extent of tensile reinforcement needs to be converted with the use of bond plates.

\section{RESEARCH METHODOLOGY}

The analysis in this research was based on secondary data supported by primary data taken from the study site, the Cibubur Transpark Project, with an emphasis on cost and time for floor slab work

To make an analysis of the cost of the work required, it must first calculate the volume of floor slab work consisting of formwork, construction, and concrete. After getting the volume of each job, it is multiplied by the unit price of each job. 
Analysis of the work time of conventional slab and metal deck composite slab is obtained from the plan for completion of work volume per day. Followed by calculating the volume of each work divided by the plan to complete the volume of work per day [5].

Table 1. Working Time Calculation Format

\begin{tabular}{|c|c|c|c|c|c|c|c|c|c|}
\hline No & $\begin{array}{c}\text { Descriptio } \\
\mathbf{n}\end{array}$ & Vol. & Unit & $\begin{array}{c}\text { Man } \\
\text { power }\end{array}$ & Index & $\begin{array}{l}\text { Productivity } \\
\text { per Day }\end{array}$ & $\begin{array}{c}\text { Number of } \\
\text { days } \\
\text { completed } \\
\text { work for } 1 \\
\text { worker }\end{array}$ & $\begin{array}{l}\text { Total } \\
\text { Man } \\
\text { power }\end{array}$ & $\begin{array}{c}\text { Workda } \\
\text { y Plan }\end{array}$ \\
\hline & & (a) & & & (b) & (c) & (d) & (e) & $(f)$ \\
\hline & \multicolumn{3}{|l|}{ Floor Slab } & & & & & & \\
\hline 1 & ${ }_{x}^{\text {Description }}$ & - & $\mathrm{m} 2$ & Worker & - & $1 / b$ & $a / c$ & - & $d / e$ \\
\hline
\end{tabular}

Source: Andriansyah, 2014 [4]

\section{RESULTS AND DISCUSSIONS}

\section{Cost Analysis of Conventional Slab Method and Metal Deck Composite Slab Method}

After getting the volume of each job, then an analysis of the price of the work unit is made so that the unit price of the work is obtained and then multiplied by the total volume of work to get the cost of the work.

Table 2. Cost of Conventional Slab

\begin{tabular}{|c|c|c|c|c|c|}
\hline No. & Work Item & Unit & Volume & Unit Price (Rp) & Total (Rp) \\
\hline & 16th Floor & & & & \\
\hline 1 & Formwork & $m^{2}$ & $1.319,37$ & $412.215,40$ & $543.865 .446,42$ \\
\hline 2 & Reinforcement & $\mathrm{kg}$ & $12.200,59$ & $13.219,28$ & $161.283 .008,60$ \\
\hline 3 & $\begin{array}{l}\text { Concrete K- } \\
350\end{array}$ & $\mathrm{~m}^{3}$ & 171,52 & $1.105 .362,75$ & $189.590 .002,49$ \\
\hline \multicolumn{5}{|c|}{ 16th Floor Cost } & $894.738 .457,52$ \\
\hline & \multicolumn{5}{|c|}{ 17th -19 th Floor } \\
\hline 1 & Formwork & $\mathrm{m}^{2}$ & $3.958,12$ & $110.700,40$ & $438.165 .016,14$ \\
\hline 2 & Reinforcement & $\mathrm{kg}$ & $36.601,77$ & $13.219,28$ & $483.849 .025,81$ \\
\hline 3 & $\begin{array}{l}\text { Concrete K- } \\
350\end{array}$ & $\mathrm{~m}^{3}$ & 514,56 & $1.105 .362,75$ & $568.770 .007,48$ \\
\hline \multicolumn{5}{|c|}{ 17th -19 th Floor Cost } & $1.490 .784 .049,43$ \\
\hline \multicolumn{5}{|c|}{ 16th - 19th Floor Total Cost } & 2.385.522.506,95 \\
\hline
\end{tabular}

Source: Author's Processed Data, 2019 
Table 3. Cost of Metal Deck Composite Slab

\begin{tabular}{|c|c|c|c|c|c|}
\hline No. & Work Item & Unit & Volume & Unit Price (Rp) & Total (Rp) \\
\hline & 16th Floor & & & & \\
\hline 1 & Formwork & $\mathrm{m}^{2}$ & $1.319,37$ & $414.821,10$ & $547.303 .333,98$ \\
\hline 2 & Reinforcement & $\mathrm{kg}$ & $6.100,29$ & $13.219,28$ & $80.641 .504,30$ \\
\hline 3 & $\begin{array}{l}\text { Concrete K- } \\
350\end{array}$ & $\mathrm{~m}^{3}$ & 138,93 & $1.105 .362,75$ & $153.567 .902,02$ \\
\hline \multicolumn{5}{|c|}{ 16th Floor Cost } & $781.512 .740,30$ \\
\hline & \multicolumn{5}{|c|}{ 17th - 19th Floor } \\
\hline 1 & Formwork & $\mathrm{m}^{2}$ & $3.958,12$ & $262.406,10$ & $1.038 .633 .763,23$ \\
\hline 2 & Reinforcement & $\mathrm{kg}$ & $18.300,88$ & $13.219,28$ & $241.924 .512,90$ \\
\hline 3 & $\begin{array}{l}\text { Concrete K- } \\
350\end{array}$ & $\mathrm{~m}^{3}$ & 416,79 & $1.105 .362,75$ & $460.703 .706,06$ \\
\hline \multicolumn{5}{|c|}{ 17th - 19th Floor Cost } & $1.741 .261 .982,19$ \\
\hline \multicolumn{5}{|c|}{ 16th - 19th Floor Total Cost } & 2.522.774.722,49 \\
\hline
\end{tabular}

Source: Author's Processed Data, 2019

From the analysis of tables 2 and 3 , the comparison between conventional slab and metal deck composite slab is as follows:

Table 4. Cost Comparison of Conventional Slab and Metal Deck Composite Slab

\begin{tabular}{|c|c|c|c|c|}
\hline No. & Work Item & Unit & Volume & Total Cost (Rp) \\
\hline \multicolumn{5}{|c|}{ 16th - 19th Floor Conventional Slab } \\
\hline 1 & Formwork & $m^{2}$ & $1.319,37$ & $982.030 .462,57$ \\
\hline 2 & Reinforcement & $\mathrm{kg}$ & $12.200,59$ & $645.132 .034,41$ \\
\hline \multirow[t]{2}{*}{3} & Concrete K-350 & $\mathrm{m}^{3}$ & 171,52 & $758.360 .009,97$ \\
\hline & \multicolumn{3}{|l|}{ Total } & 2.385.522.506,95 \\
\hline \multicolumn{5}{|c|}{ 16th - 19th Floor Metal Deck Composite Slab } \\
\hline 1 & Formwork & $m^{2}$ & $1.319,37$ & 1.585.937.097,21 \\
\hline 2 & Reinforcement & $\mathrm{kg}$ & $6.100,29$ & $322.566 .017,20$ \\
\hline \multirow[t]{2}{*}{3} & Concrete K-350 & $\mathrm{m}^{3}$ & 138,93 & $614.271 .608,08$ \\
\hline & \multicolumn{3}{|l|}{ Total } & 2.522.774.722,49 \\
\hline \multicolumn{5}{|c|}{ Cost efficiency of metal deck slab over conventional slab } \\
\hline 1 & Formwork & & & $603.906 .634,64$ \\
\hline 2 & Reinforcement & & & $322.566 .017,20$ \\
\hline \multirow[t]{2}{*}{3} & Concrete K-350 & & & $144.088 .401,89$ \\
\hline & \multicolumn{3}{|l|}{ Total } & $137.252 .215,54$ \\
\hline
\end{tabular}

Source: Author's Processed Data, 2019 
From the table 4 , it is obtained that the total estimated cost of conventional slab and composite metal deck has a cost difference of Rp. 137.252.215,54 and conclude that the work of metal deck composite slab is more expensive when compared to conventional slab.

\section{Cost Analysis of Conventional Slab Method and Metal Deck Composite Slab Method}

Work time for conventional floor slab and metal deck composite floor slab is obtained from the planned work volume completion per day. As for the completion of the volume of work per day it is assumed as follows:

Table 5. Manpower Plan

\begin{tabular}{|r|l|l|r|r|r|}
\hline No & Description & Tenaga Kerja & Index & $\begin{array}{c}\text { Productivity } \\
\text { per Day }\end{array}$ & $\begin{array}{c}\text { Total } \\
\text { Man } \\
\text { power }\end{array}$ \\
\hline 1 & Conventional & Worker & \multicolumn{1}{c|}{$\mathrm{a}$} & \multicolumn{1}{c|}{$\mathrm{b}=1 / \mathrm{a}$} & \multicolumn{1}{c|}{$\mathrm{c}$} \\
\hline & Formwork & Carpenter & 0,330 & 1,52 & 66 \\
\hline & & Head Worker & 0,033 & 30,03 & 33 \\
\hline & & Foreman & 0,033 & 30,30 & 3 \\
\hline 2 & Reinforcement & Worker & 0,070 & 14,29 & 70 \\
\hline & & Reinforcement & 0,070 & 14,29 & 70 \\
\hline & & Worker & 0,007 & 142,86 & 7 \\
\hline & & Fead Worker & 0,004 & 250,00 & 4 \\
\hline 3 & Concrete K-350 & Worker & 0,438 & 2,29 & 14 \\
\hline & & Foreman & 0,031 & 32,00 & 1 \\
\hline 4 & Metal Deck & Worker & 0,440 & 2,27 & 66 \\
\hline & & Carpenter & 0,220 & 4,55 & 33 \\
\hline & & Head Worker & 0,022 & 45,45 & 3 \\
\hline & & Foreman & 0,022 & 45,45 & 3 \\
\hline
\end{tabular}

Source: SNI 7394:2008 No.6.24 [3] and Author's Processed Data

From table 11, it is continued by calculating the volume of each work divided by the planned volume of work completion per day. From the results of these calculations obtained work plan for each work as follows: 
Table 6. Work Time Calculation of Conventional Slab

\begin{tabular}{|c|c|c|c|c|c|c|c|c|c|}
\hline No & Description & Vol. & Unit & $\begin{array}{l}\text { Man } \\
\text { power }\end{array}$ & Index & $\begin{array}{c}\text { Productivity } \\
\text { per Day }\end{array}$ & $\begin{array}{c}\text { Number of } \\
\text { days } \\
\text { completed } \\
\text { work for } 1 \\
\text { worker }\end{array}$ & $\begin{array}{l}\text { Total } \\
\text { Man } \\
\text { power }\end{array}$ & $\begin{array}{c}\text { Workday } \\
\text { Plan }\end{array}$ \\
\hline & & $\mathrm{a}$ & & & $b$ & $c=1 / b$ & $\mathrm{~d}=\mathrm{a} / \mathrm{b}$ & $\mathrm{e}$ & $f=e / d$ \\
\hline & \multicolumn{3}{|c|}{ 16th - 19th Floor } & & & & & & \\
\hline \multirow[t]{4}{*}{1} & Conventional & 5277,49 & $\mathrm{~m} 2$ & Worker & 0,660 & 1,52 & 3483,14 & 66 & 52,77 \\
\hline & Formwork & & & Carpenter & 0,330 & 3,03 & 1741,57 & 33 & 52,77 \\
\hline & & & & $\begin{array}{l}\text { Head } \\
\text { Worker }\end{array}$ & 0,033 & 30,30 & 174,16 & 3 & 52,77 \\
\hline & & & & Foreman & 0,033 & 30,30 & 174,16 & 3 & 52,77 \\
\hline \multirow[t]{4}{*}{2} & Reinforcement & $48.802,36$ & $\mathrm{~kg}$ & Worker & 0,070 & 14,29 & 341,62 & 70 & 4,88 \\
\hline & & & & $\begin{array}{l}\text { Reinforce } \\
\text { Ment } \\
\text { Worker }\end{array}$ & 0,070 & 14,29 & 341,62 & 70 & 4,88 \\
\hline & & & & $\begin{array}{l}\text { Head } \\
\text { Worker }\end{array}$ & 0,007 & 142,86 & 34,16 & 7 & 4,88 \\
\hline & & & & Foreman & 0,004 & 250,00 & 19,52 & 4 & 4,88 \\
\hline \multirow[t]{7}{*}{3} & Concrete & 686,07 & m3 & Worker & 0,438 & 2,29 & 300,16 & 14 & 21,44 \\
\hline & & & & Foreman & 0,031 & 32,00 & 21,44 & 1 & 21,44 \\
\hline & & & & & & & & & \\
\hline & Formwork & & & & & & & & 52,77 \\
\hline & Reinforcement & & & & & & & & 4,88 \\
\hline & Concrete & & & & & & & & 21,44 \\
\hline & Total & & & & & & & & 80 \\
\hline
\end{tabular}

Source: Author's Processed Data, 2019

Table 7. Work Time Calculation of Metal Deck Composite Slab

\begin{tabular}{|c|c|c|c|c|c|c|c|c|c|}
\hline No & Description & Vol. & Unit & $\begin{array}{l}\text { Man } \\
\text { power }\end{array}$ & Index & $\begin{array}{l}\text { Productivity } \\
\text { per Day }\end{array}$ & $\begin{array}{c}\text { Number of } \\
\text { days } \\
\text { completed } \\
\text { work for } 1 \\
\text { worker }\end{array}$ & $\begin{array}{l}\text { Total } \\
\text { Man } \\
\text { power }\end{array}$ & $\begin{array}{c}\text { Workday } \\
\text { Plan }\end{array}$ \\
\hline & & a & & & b & $\mathrm{c}=1 / \mathrm{b}$ & $\mathrm{d}=\mathrm{a} / \mathrm{b}$ & e & $\mathrm{f}=\mathrm{e} / \mathrm{d}$ \\
\hline & \multicolumn{3}{|c|}{ 16th - 19th Floor } & & & & & & \\
\hline \multirow[t]{4}{*}{1} & Metal Deck & 5277,49 & $\mathrm{~m} 2$ & Worker & 0,440 & 2,27 & 2322,09 & 66 & 35,18 \\
\hline & & & & Carpenter & 0,220 & 4,55 & 1161,05 & 33 & 35,18 \\
\hline & & & & $\begin{array}{l}\text { Head } \\
\text { Worker }\end{array}$ & 0,022 & 45,45 & 116,10 & 3 & 35,18 \\
\hline & & & & Foreman & 0,022 & 45,45 & 116,10 & 3 & 35,18 \\
\hline \multirow[t]{2}{*}{2} & Reinforcement & $24.401,18$ & $\mathrm{~kg}$ & Worker & 0,070 & 14,29 & 170,81 & 70 & 2,44 \\
\hline & & & & $\begin{array}{l}\text { Reinforce } \\
\text { ment } \\
\text { Worker }\end{array}$ & 0,070 & 14,29 & 170,81 & 70 & 2,44 \\
\hline
\end{tabular}




\begin{tabular}{|c|c|c|c|c|c|c|c|c|c|}
\hline & & & & $\begin{array}{l}\text { Head } \\
\text { Worker }\end{array}$ & 0,007 & 142,86 & 17,08 & 7 & 2,44 \\
\hline & & & & Foreman & 0,004 & 250,00 & 9,76 & 4 & 2,44 \\
\hline \multirow[t]{6}{*}{3} & Concrete & 555,72 & m3 & Worker & 0,438 & 2,29 & 243,13 & 14 & 17,37 \\
\hline & & & & Foreman & 0,031 & 32,00 & 17,37 & 1 & 17,37 \\
\hline & & & & & & & & & \\
\hline & Metal deck & & & & & & & & 35,18 \\
\hline & Reinforcement & & & & & & & & 2,44 \\
\hline & Concrete & & & & & & & & 17,37 \\
\hline & Total & & & & & & & & 55 \\
\hline
\end{tabular}

Source: Author's Processed Data, 2019

From the results of the calculation of the work time above, there are difference time. For conventional floor slab takes 80 days, whereas for metal deck composite floor slab work takes 55 days. Therfore metal deck composite slab method is 25 days faster than conventional slab method.

\section{CONCLUSIONS}

Table 8. Cost and Time Comparison

\begin{tabular}{|c|l|c|c|c|}
\hline No & Description & $\begin{array}{c}\text { 16th - 19th Floor } \\
\text { Conventional Slab }\end{array}$ & $\begin{array}{c}\text { 16th - 19th Floor } \\
\text { Metal Deck Slab }\end{array}$ & $\begin{array}{c}\text { Cost and Time } \\
\text { Efficiency }\end{array}$ \\
\hline 1 & Total Cost & Rp2.385.522.507 & Rp2.522.774.722 & Rp137.252.216 \\
\hline 2 & Work Time & 80 days & 55 days & 25 days \\
\hline
\end{tabular}

Source: Author's Processed Data, 2019

Based on Table 8 and from the analysis and discussion in the previous chapter, we get the following conclusions

1) In the Cibubur Transpark Project Tower $C$ area for metal deck composite floor slab method is more expensive than conventional floor slab method with a difference of Rp. $137.252 .215,54$

2) In the Cibubur Transpark Project Tower $\mathrm{C}$ area, the work time of metal deck composite floor slab is faster than conventional floor slab with a work time difference of 25 days.

\section{REFERENCE}

[1] Afriyono, H. (2019): Efisiensi Pelat Beton dengan Bekisting dan Tulangan Konvensional menjadi Floordeck dan Tulangan Wiremesh. Buletin Profesi Insinyur, Banjarmasin.

[2] Andriansyah. (2014): Analisa Perbandingan Biaya dan Waktu Pekerjaan Plat Lantai Konvensional Dan Plat Lantai Komposit Metal Deck Studi Kasus Proyek Pembangunan Gedung PGN Area Palembang. Universitas Mercubuana, Jakarta.

[3] Anonim, SNI 7394-2008. (2008): Tata Cara Perhitungan Harga Satuan Pekerjaan Beton Untuk Konstruksi Bangunan Gedung dan Perumahan. Badan Standarisasi Nasional, Jakarta.

[4] Fastaria, R., \& Putri, Y. E. (2014): Analisa Perbandingan Metode Halfslab dan Plat Komposit Bondek Pekerjaan Struktur Plat Lantai Proyek Pembangunan Apartement De Papilio Tamansari Surabaya. Jurnal Teknik ITS, Surabaya.

[5] Leneldo, A., \& Taufik, H. (2016): Perbandingan Harga Plat Lantai Ruko Antara Plat Lantai Konvensional Dan Plat Lantai Steel Decking (Case Study: Area Pekanbaru). Jurnal 
Fakultas Teknik, Pekanbaru.

[6] Naray, Farly. (2015): Analisa Perencanaan Dan Pelaksanaan Pelat Bondek Sebagai Pengganti Tulangan Tarik Konstruksi Pelat Lantai Pada Proyek Pembangunan Gedung Kuliah Terpadu Politeknik Negeri Manado. Politeknik Negeri Manado, Manado. 